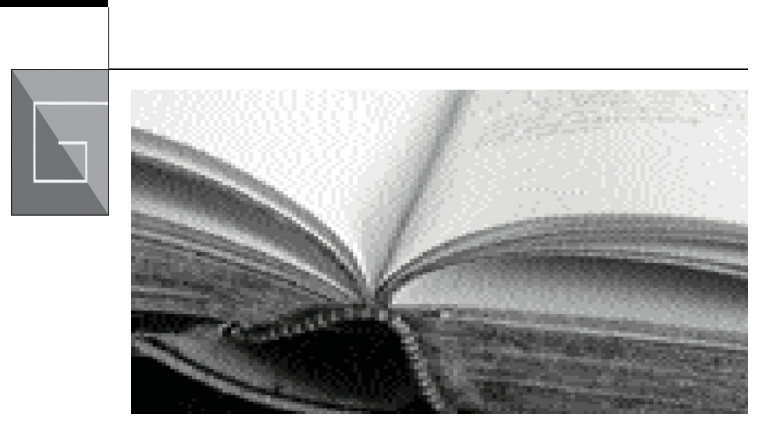

\title{
L'enseignement de la gestion en France : identité, défis et enjeux
}

\author{
Sous la direction d'Alain Burlaud et Frank Bournois \\ Editions Management \& Société, avril 2021, 452 p., 25 euros
}

A llons immédiatement droit au but : la lecture de l'ouvrage coordonné par Alain Burlaud et Frank Bournois, avec le soutien d'un conseil scientifique de renom, va très vite s'imposer comme un passage obligé pour tous les enseignantschercheurs en sciences de gestion et du management, quelle que soit leur institution d'appartenance, quelle que soit leur discipline, quelle que soit leur génération. En effet, ayant fait appel à de remarquables expertises, nos deux collègues donnent à voir une brillante plongée dans l'enseignement de la gestion en France, en adoptant à la fois un regard rétrospectif indispensable pour comprendre aujourd'hui et demain, et une réflexion de haute tenue sur des enjeux contemporains majeurs, notamment au moment même où une révolution est en œuvre dans le recrutement des professeurs des universités, comme nous avons eu l'occasion de le souligner récemment ${ }^{1}$. Chacune des contributions conduit le lecteur au cœur de processus décisionnels et politiques, et met en lumière des jeux d'acteurs, tout particulièrement dans l'institutionnalisation des sciences de gestion et du management dont on peut imaginer que les jeunes enseignants-chercheurs (disons, les moins de quarante ans !) ignorent presque tout.

L'ouvrage est très astucieusement construit en trois parties. En fait, il comprend deux parties principales auxquelles est associée une troisième partie réflexive, rédigée par 
Alain Burlaud et Frank Bournois, qui propose une synthèse sur le mode du « d'où venons-nous et où allons-nous ? » Les deux parties principales abordent, dans un premier temps, la construction académique des sciences de gestion et du management sous différentes facettes, puis dans un second temps, quelques tendances se dessinant depuis une quinzaine d'années, là aussi à travers plusieurs « instantanés » relatifs à l'ingénierie pédagogique, à la recherche, à la gouvernance des universités, mais aussi à la question (cruelle) de l'endettement des étudiantes et étudiants. L'une des forces principales de l'ouvrage est de laisser la parole à des acteurs clé dont la légitimité est incontestable et incontestée, qui plus est des acteurs ayant été souvent des moteurs de la « révolution managériale » à l'université et dans les business schools (notamment Bernard Dizambourg et Alain Strazzieri pour les IUT, Maurice A. Saias pour les IAE, et Geneviève Causse pour le développement des enseignements en alternance). Qu'il soit ici permis d'avoir une pensée émue pour Bertrand Moingeon et Jacques Rojot, impliqués dans le projet conduit par Alain Burlaud et Frank Bournois, mais qui n'ont pas pu, hélas, voir sa concrétisation.

N'ayons pas peur de l'écrire : la lecture de la première partie de l'ouvrage est un « régal » de tous les instants. En effet, nous sommes plongés avec délectation au temps des pionniers, ces Maîtres auxquels nous devons aujourd'hui l'institutionnalisation progressive des sciences de gestion et du management. Avec des dates marquantes : la création des IAE en 1955 sous l'impulsion de Gaston Berger et Pierre Tabatoni, la création des IUT en 1966, l'expérience « révolutionnaire » en 1968 de la création du centre expérimental de Dauphine sous l'impulsion d'Edgar Faure ${ }^{2}$ et, bien sûr, le rôle central tenu par la Fnege au début des années 1970 dans la création ex nihilo d'un corps professoral en sciences de gestion et du management, après un séjour souvent inoubliable - avec ou sans épouse (!) - en Amérique du Nord en vue de décrocher un $\mathrm{PhD}$ en management. «Épouse » car Geneviève Causse note avec son humour habituel que les femmes n'étaient pas alors vraiment présentes dans les listes successives des happy fews de la FNEGE. Autres temps, autres mœurs ! D'autres thématiques sont évidemment abordées dans les huit chapitres et méritent une lecture approfondie. Citons par exemple la question brûlante de l'impact managérial des recherches conduites au sein de notre communauté, qui interpelle manifestement Michel Kalika et Maurice Thévenet, sans doute dubitatifs face aux dérives de publications ésotériques et «hors sol » dans des supports prestigieux... totalement ignorés par les décideurs en entreprise.

La deuxième partie de l'ouvrage opte pour une tonalité différente, mais complémentaire : les sciences de gestion et du management sont sous la menace de puissantes forces centrifuges. Est-ce tomber dans une analyse «idéologique » que de l'affirmer? Certainement pas. Les onze chapitres choisissent des angles d'attaque singuliers pour identifier de vrais dangers dont il faut prendre conscience, qu'il s'agisse des modalités « disputables » de constitution du corps professoral dans les prochaines années, du potentiel

2. Le Pen C., éd. (1995), Paris-Dauphine : vingt-cinq ans de sciences d'organisation, Masson, Paris. 
«cauchemar» en pédagogie sous les coups de boutoir du numérique (que la crise de la Covid-19 a d'ailleurs mis sur le devant de la scène), sur le diktat insupportable des classements de revues conduisant à un appauvrissement de la pensée francophone, ou encore sur les ravages du New Public Management dans les universités françaises, au bord de l'asphyxie financière pour certaines. Nous ne résistons pas à la tentation d'évoquer, sous forme de clin d'œil, l'étonnement d'un collègue renommé en visite à Harvard lorsqu'il découvrit que la dean's house exposait... un vrai Picasso au bas d'un escalier! En paraphrasant une vieille publicité télévisée, nous pourrions dire: «France, États-Unis : nous n'avons pas les mêmes valeurs ! »

En refermant l'ouvrage coordonné par Alain Burlaud et Frank Bournois, le lecteur sort enrichi au-delà de ses espérances. Plongé dans un passé pas si lointain (à peine plus de cinquante ans), narré brillamment par certaines et certains de ses protagonistes les plus marquants, il comprend beaucoup mieux les enjeux actuels et surtout futurs de l'enseignement de la gestion en France. Alors, sans hésitation, il faut s'immerger dans les contributions réunies pour l'occasion, que l'on soit doctorant, ou enseignantchercheur débutant... ou confirmé. Chacune et chacun d'entre nous, dans notre « communauté de destin » des sciences de gestion et du management, y trouvera des réflexions stimulantes qui ne le laisseront certainement pas insensible, bien au contraire, pour penser l'avenir.

Gilles PACHÉ Aix-Marseille Université 